\title{
Mathematical modeling of the impact of treatment on the dynamics of typhoid
}

\author{
Halson O. Nyaberi ${ }^{*}$ and Jane S. Musaili²
}

\section{*Correspondence:}

halsonogeto@gmail.com

${ }^{1}$ Department

of Mathematics, The

Technical University of Kenya,

Nairobi, Kenya

Full list of author information

is available at the end of the

article

\begin{abstract}
In this paper, we propose a mathematical model for the transmission of typhoid which analyzes the impact of treatment of the infected individuals on the dynamics of the disease. The model consists of human population and pathogen population. The human population is subdivided into three compartments, namely susceptible individuals, infected individuals, and recovered individuals and pathogen population comprises one compartment. We derived the basic reproduction number $\mathcal{R}_{0}$, and analyzed the dynamical behaviors of both disease free equilibrium and endemic equilibrium by the theory of ordinary differential equations. Using MATLAB, we carried out numerical simulation and the findings indicate that effective treatment is adequate in eradicating typhoid fever.
\end{abstract}

Keywords: Typhoid, Basic reproduction number, Stability

\section{Introduction}

Typhoid fever is an infection that is caused by a bacterium called Salmonella Typhi. The bacteria enter the body system through the consumption of contaminated food or water. Typhoid fever is characterized by prolonged fever, headache, nausea, loss of appetite, constipation, and diarrhea. When these symptoms are severe, typhoid fever may lead to death. Each year it is estimated that an average of 16 million cases and an average of 145000 typhoid-related deaths are recorded worldwide [1]

Typhoid fever can be treated with antibiotics. According to [1], the disease can be prevented through vaccination with the following vaccines recommended: an injectable typhoid conjugate vaccine (TCV); an injectable polysaccharide vaccine based on the purified Vi antigen and it is for children aged 2 years and above; an oral live attenuated Ty2Ia vaccine in capsules and it is suitable for children aged over 6 years. Among the above 3 vaccines, (TCV) is mostly preferred since it can be used for all ages including infants to adults and it has an improved immunological property.

In Kenya, typhoid is a public health burden for children aged below 15 years. They are at a higher risk of contracting the disease, because they are prone to playing with rainwater. The global burden of diseases shows that in 2016, Kenya had 97,767 typhoid cases of which $62 \%$ belonged to children aged less than 15 years and 1075 typhoid cases deaths with $66 \%$ among children aged less than 15 years [2].

(c) The Author(s), 2021. Open Access This article is licensed under a Creative Commons Attribution 4.0 International License, which permits use, sharing, adaptation, distribution and reproduction in any medium or format, as long as you give appropriate credit to the original author(s) and the source, provide a link to the Creative Commons licence, and indicate if changes were made. The images or other third party material in this article are included in the article's Creative Commons licence, unless indicated otherwise in a credit line to the material. If material is not included in the article's Creative Commons licence and your intended use is not permitted by statutory regulation or exceeds the permitted use, you will need to obtain permission directly from the copyright holder. To view a copy of this licence, visit http:// creativecommons.org/licenses/by/4.0/. 
According to CDC [3], typhoid fever cases are high in Kenyan slums, such as Kibera in the outskirts of Nairobi, because it is overpopulated and has inadequate sanitation facilities for containing and disposing of human wastes.

Several mathematical models have been formulated to describe the dynamics of the spread of typhoid fever. For instance, Nthiiri et al. [4] formulated a model that aimed at studying the dynamics of typhoid fever using protection as a control strategy and from the numerical simulation results, they found out that increasing the protection would significantly reduce the disease prevalence in a population.

Stephen and Nyerere [5] came up with a model for accessing the impact of educational campaigns in controlling the transmission of typhoid in the community. From the analysis of the model, they established that creating awareness was a possible way of curbing transmission by more than $40 \%$.

Many scholars have developed mathematical models that aim at studying the dynamics of typhoid fevers while incorporating a combination of control strategies, such as vaccination, treatment, and screening [6]. In this study, we investigate the impact of treatment alone in controlling typhoid fever. The paper is organized as follows: in this section, we have provided background information of typhoid fever and given a brief discussion on previous works on the disease and other findings on a general impact of control strategies on epidemics; in "Methods" section, we have formulated and described the mathematical model. In "Analysis of the model" section, we presented the model's basic properties, and determined its equilibrium points, the basic reproduction number $\mathcal{R}_{0}$, and the existence and uniqueness of the disease positive endemic equilibrium. We have also established the sufficient conditions for the local and global stabilities of the disease-free and the disease-endemic equilibria and proved that they are both globally asymptotically stable. "Results" section presents numerical simulations to verify our theoretical results. Finally, we conclude this work in "Conclusion" section.

\section{Methods}

In this study, we develop a model with pathogen population, $N_{\mathrm{B}}$ denoted by $\mathrm{B}(t)$ and human population, $N_{\mathrm{H}}$. That is, the total population will be $N(t)=N_{\mathrm{H}}(t)+N_{\mathrm{P}}(t)$. The human population is subdivided into Susceptible individuals-S(t), Infected individuals$\mathrm{I}(\mathrm{t})$ and Recovered individuals $-\mathrm{R}(\mathrm{t})$ compartments. The model assumes that human population will be recruited to susceptible compartment at the rate $\Lambda$ and susceptible individuals are infected at the rate of $\frac{\alpha B}{\kappa+B}$ where $\alpha$ is the rate of Salmonella Typhi ingestion in drinks or foods and $\frac{B}{\kappa+B}$ is the probability of individuals in consuming foods or drinks contaminated with typhoid causing bacteria. All human populations experience natural death at the rate $\mu$. In addition, the infected individuals die from typhoid at the rate $\delta$. Infected individuals are treated at the rate $\epsilon$. Infected individuals will excrete Salmonella Typhi bacteria to the environment at the rate $\eta$ and Salmonella Typhi will die at the rate $v$ Finally, $0 \leq \omega \leq 1$ ) is a constant representing the treatment of infected individuals. This parameter changes the rate of infection as well as the rate shedding of Salmonella Typhi to the environment.

\section{Assumptions of the model}

The following are the assumptions of the model: 
(i) The population birth and death rate occur at different rates.

(ii) Typhoid is transmitted through ingestion Salmonella bacteria in foods or drinks.

(iii) Infectious individuals recover as a result of treatment.

(iv) There is permanent immunity upon recovery.

(v) All the newly born individuals will join only susceptible class.

(vi) Infectious individuals excrete Salmonella Typhi bacterium to the environment.

\section{Model flowchart and equations}

From Fig. 1 we have the following equations of the model:

$$
\begin{aligned}
& \frac{\mathrm{dS}}{\mathrm{dt}}=\Lambda-\left[\frac{(1-\omega) \alpha B}{\kappa+B}+\mu\right] S \\
& \frac{\mathrm{dI}}{\mathrm{dt}}=\frac{(1-\omega) \alpha B S}{\kappa+B}-(\mu+\epsilon+\delta) I \\
& \frac{\mathrm{dR}}{\mathrm{dt}}=\epsilon I-\mu R \\
& \frac{\mathrm{dB}}{\mathrm{dt}}=(1-\omega) \eta I-\nu B
\end{aligned}
$$

\section{Analysis of the model}

Since system (1) describes human population and pathogen population, all feasible solutions are uniformly bounded in a proper subset of $\Gamma=\Gamma_{\mathrm{H}} \times \Gamma_{\mathrm{B}}$. The feasible region

$$
\Gamma_{\mathrm{H}}=\left\{(S, I, R) \in \mathbb{R}_{+}^{3} ; S>0, I, R \geq 0 ; N_{\mathrm{H}} \leq \frac{\Lambda}{\mu}\right\} \cup \Gamma_{\mathrm{B}}=\left\{\mathrm{B} \in \mathbb{R}_{+} ; \mathrm{B} \geq 0 ; N_{\mathrm{B}} \leq \frac{\Lambda(1-\omega) \eta}{\mu \nu}\right\}
$$

is positively invariant.

\section{Disease free equilibrium (DFE)}

Let the DFE be denoted by $E_{0}\left(S_{0}, I_{0}, R_{0}, B_{0}\right)$. To determine the disease free equilibrium point, we equate the right-hand side of system (1) to zero and substitute $S=S_{0}$, $I=I_{0}=0, R=R_{0}=0$ and $B=B_{0}=0$. Solving the remaining equation we get $S_{0}=\frac{\Lambda}{\mu}$. Thus

$$
E_{0}\left(S_{0}, I_{0}, R_{0}, B_{0}\right)=E_{0}\left(\frac{\Lambda}{\mu}, 0,0,0\right)
$$

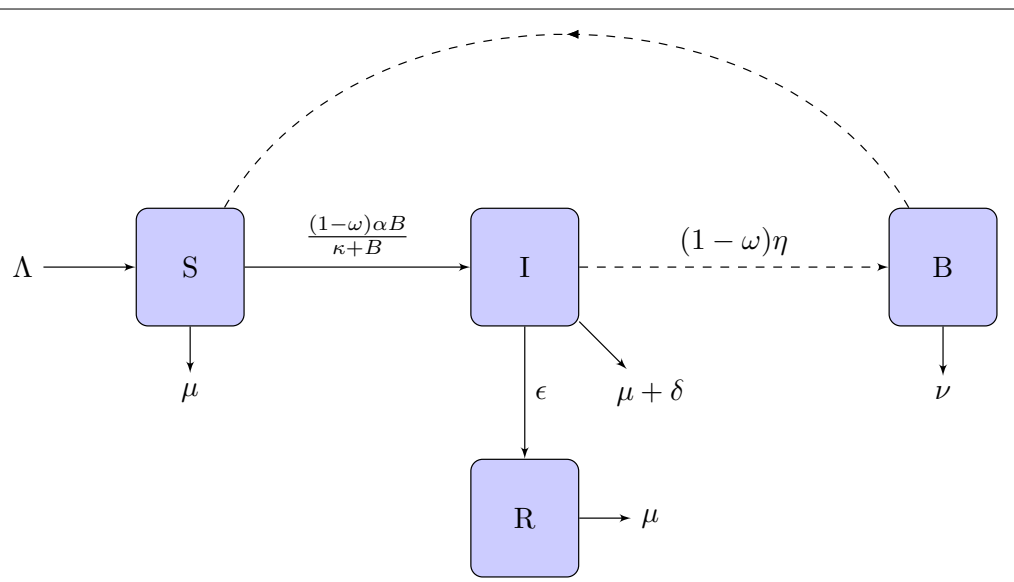

Fig. 1 Flowchart 


\section{The basic reproduction number}

The basic reproduction number $\left(\mathcal{R}_{0}\right)$ is the expected number of secondary infections produced in a completely susceptible population by a typical infected individual during his/her infectious lifetime in the presence of protection. By using the next generation matrix approach [7], $\mathcal{R}_{0}$ is given by $\rho\left(F V^{-1}\right)$ (the spectral radius of the matrix $\left.F V^{-1}\right)$. The matrices $F$ and $V$ are given by

$$
F=\left[\begin{array}{cc}
0 & \frac{(1-\omega) \Lambda \alpha}{\mu \kappa} \\
0 & 0
\end{array}\right]
$$

and

$$
V=\left[\begin{array}{cc}
(\mu+\epsilon+\delta) & 0 \\
-(1-\omega) \eta & v
\end{array}\right]
$$

Therefore, it follows that

$$
\mathcal{R}_{0}=\rho\left(F V^{-1}\right)=\frac{(1-\omega)^{2} \eta \Lambda \alpha}{\nu \mu \kappa(\mu+\epsilon+\delta)}
$$

\section{Existence of the endemic equilibrium (EE)}

We denote our endemic equilibrium point as $E^{*}\left(S^{*}, I^{*}, R^{*}, B^{*}\right)$.

Theorem 1 There exists a unique endemic equilibrium of system (1) when $\mathcal{R}_{0}>1$.

Proof To prove this theorem, we equate the right-hand side of system (1) to zero and replace $S, I, R$ and $B$ with $S^{*}, I^{*}, R^{*}$, and $B^{*}$, respectively, to get

$$
\begin{aligned}
& 0=\Lambda-\left[\frac{(1-\omega) \alpha B^{*}}{\kappa+B^{*}}+\mu\right] S^{*} \\
& 0=\frac{(1-\omega) \alpha B^{*} S^{*}}{\kappa+B^{*}}-(\mu+\epsilon+\delta) I^{*} \\
& 0=\epsilon I^{*}-\mu R^{*} \\
& 0=(1-\omega) \eta I^{*}-\nu B^{*}
\end{aligned}
$$

From the last two equations of system (2) we have

$$
\begin{aligned}
R^{*} & =\frac{\epsilon I^{*}}{\mu}, \\
B^{*} & =\frac{(1-\omega) \eta I^{*}}{v} .
\end{aligned}
$$

Adding the first and the second equation of system (2) the solving for $S^{*}$ we obtain

$$
S^{*}=\frac{\Lambda-(\mu+\epsilon+\delta) I^{*}}{\mu} .
$$


Now we substitute Eqs. (4) and (5) in the second equation of system (2) and then solve for $I^{*}$ to obtain

$$
\begin{aligned}
I^{*} & =\frac{\kappa(\mu+\epsilon+\delta)\left(1-\frac{(1-\omega)^{2} \Lambda \alpha \eta}{\nu \mu \kappa(\mu+\epsilon+\delta)}\right)}{-\left(\frac{(1-\omega)^{2}(\mu+\epsilon+\delta) \alpha \eta}{\nu \mu}+\frac{(1-\omega)(\mu+\epsilon+\delta) \eta}{\nu}\right)} \\
& =\frac{-\kappa \nu \mu\left(1-\mathcal{R}_{0}\right)}{(1-\omega)^{2} \alpha \eta+(1-\omega) \mu \eta},
\end{aligned}
$$

and it follows that $I^{*}>0$ given that $\mathcal{R}_{0}>1$.

\section{Local stability of disease free equilibrium point}

In this section, we analyze the local stability of the disease free equilibrium point.

Theorem 2 The disease-free equilibrium (DFE) is locally asymptotically stable if and only if all eigenvalues of the Jacobian matrix of system (1) at the DFE have a negative real part.

Proof The Jacobian of system at the DFE is given by

$$
J\left(E_{0}\right)=\left[\begin{array}{cccc}
-\mu & 0 & 0 & -\frac{(1-\omega) \alpha \Lambda}{\kappa \mu} \\
0 & -(\mu+\epsilon+\delta) & 0 & \frac{(1-\omega) \alpha \Lambda}{\kappa \mu} \\
0 & \epsilon & -\mu & 0 \\
0 & (1-\omega) \eta & 0 & -v
\end{array}\right]
$$

From matrix (7) we obtain the characteristic equation

$$
(\lambda+\mu)^{2}(\lambda+v)[\lambda+(\mu+\epsilon+\delta)]=0
$$

Solving for $\lambda$ in Eq. (8) we get

$\lambda_{1}=\lambda_{2}=-\mu, \lambda_{3}=-v, \lambda_{4}=-(\mu+\epsilon+\delta)$

Clearly, all eigenvalues have a negative real part as required.

\section{Global stability of disease free equilibrium point}

In this section, we analyze the global stability of the disease free equilibrium point by following [8].

Theorem 3 The disease free equilibrium is globally asymptotically stable if $\mathcal{R}_{0} \leq 1$. 
Proof We begin the proof by constructing the Lyapunov function

$$
V=(1-\omega) \eta I+(\mu+\epsilon+\delta) B .
$$

Differentiating Eq. (9) with respect to $t$ we get

$$
\frac{\mathrm{dV}}{\mathrm{dt}}=(1-\omega) \eta \frac{\mathrm{dI}}{\mathrm{dt}}+(\mu+\epsilon+\delta) \frac{\mathrm{dB}}{\mathrm{dt}} .
$$

Now we plug in values $\frac{\mathrm{dI}}{\mathrm{dt}}$ and $\frac{\mathrm{dB}}{\mathrm{dt}}$ into Eq. (10) using system (1) to get

$$
\begin{aligned}
\frac{\mathrm{dV}}{\mathrm{dt}} & =(1-\omega) \eta\left(\frac{(1-\omega) \alpha B S}{\kappa+B}-(\mu+\epsilon+\delta) I\right)+(\mu+\epsilon+\delta)((1-\omega) \eta I-v B) \\
& =\left(\frac{(1-\omega)^{2} \eta \alpha S}{(\kappa+B)(\mu+\epsilon+\delta) \nu}-1\right)(\mu+\epsilon+\delta) \nu B
\end{aligned}
$$

Substituting $S=S_{0}=\frac{\Lambda}{\mu}$ in Eq. (11) we obtain

$$
\begin{aligned}
\frac{\mathrm{dV}}{\mathrm{dt}} & =\left(\frac{(1-\omega)^{2} \eta \Lambda \alpha}{\mu(\kappa+B)(\mu+\epsilon+\delta) \nu}-1\right)(\mu+\epsilon+\delta) \nu B \\
& \leq\left(\frac{(1-\omega)^{2} \eta \Lambda \alpha}{\mu \kappa(\mu+\epsilon+\delta) \nu}-1\right)(\mu+\epsilon+\delta) \nu B .
\end{aligned}
$$

And it follows that

$$
\frac{\mathrm{dV}}{\mathrm{dt}} \leq\left(\mathcal{R}_{0}-1\right)(\mu+\epsilon+\delta) \nu B
$$

Clearly $\frac{\mathrm{dV}}{\mathrm{dt}} \leq 0$ if $\mathcal{R}_{0} \leq 1$. Moreover, $\frac{\mathrm{dV}}{\mathrm{dt}}=0 \Leftrightarrow \mathcal{R}_{0}=1$ or $B=0$ which leads to $I=R=0$. Thus, it follows that the invariant set of system (1) on the set $\left\{(S, I, R, B) \in \Gamma:\left.\dot{V}\right|_{(1)} \leq 0\right\}$ is the singleton the disease free equilibrium point $\left(E_{0}\right)$. Hence, from the LaSalle's invariance principle [8], $E_{0}$ is globally asymptotically stable on the set $\Gamma$ if $\mathcal{R}_{0} \leq 1$.

\section{Local stability of endemic equilibrium point}

In this section, we analyze the local stability of the endemic equilibrium point.

Theorem 4 The endemic equilibrium is locally asymptotically stable if $\mathcal{R}_{0}>1$.

Proof The Jacobian of system at the EE is given by

$$
J\left(E^{*}\right)=\left[\begin{array}{cccc}
-\left[\frac{(1-\omega) \alpha B^{*}}{\kappa+B^{*}}+\mu\right] & 0 & 0 & -\frac{(1-\omega) \alpha \kappa}{\left(\kappa+B^{*}\right)^{2}} S^{*} \\
\frac{(1-\omega) B^{*}}{\kappa+B^{*}} & -(\mu+\epsilon+\delta) & 0 & \frac{(1-\omega) \alpha \kappa}{\left(\kappa+B^{*}\right)^{2}} S^{*} \\
0 & \epsilon & -\mu & 0 \\
0 & (1-\omega) \eta & 0 & -v
\end{array}\right]
$$

The trace of the Jacobian matrix (13) is negative and the determinant is given by 


$$
\begin{aligned}
\operatorname{Det}\left(J\left(E^{*}\right)\right)= & -\frac{(1-\omega)^{2} \eta \Lambda \alpha \kappa \mu}{\left(\kappa+B^{*}\right)^{2}}+\frac{(1-\omega)^{2} \eta \alpha \kappa \mu}{\left(\kappa+B^{*}\right)^{2}}(\mu+\epsilon+\delta) I^{*} \\
& +\left(\frac{(1-\omega) \alpha B^{*}}{\kappa+B^{*}}+\mu\right)(\mu+\epsilon+\delta) \nu,
\end{aligned}
$$

which is positive if

$$
\frac{(1-\omega)^{2} \eta \Lambda \alpha \kappa \mu}{\left(\kappa+B^{*}\right)^{2}}<\frac{(1-\omega)^{2} \eta \alpha \kappa \mu}{\left(\kappa+B^{*}\right)^{2}}(\mu+\epsilon+\delta) I^{*}+\left(\frac{(1-\omega) \alpha B^{*}}{\kappa+B^{*}}+\mu\right)(\mu+\epsilon+\delta) \nu .
$$

Since there exist a unique endemic equilibrium of system (1) provided that $\mathcal{R}_{0}>1$ (Ref Theorem 1), if the determinant is positive, by Routh-Hurwitz criteria, the endemic state $E^{*}\left(S^{*}, I^{*}, R^{*}, B^{*}\right)$ is locally asymptotically stable.

\section{Global stability of the endemic equilibrium point}

We study the global asymptotic stability of the endemic equilibrium using LaSalle's invariance principle [8].

Theorem 5 The Endemic Equilibrium Point $E^{*}$ of system (1) is globally asymptotically stable if $R_{0}>1$.

Proof

We apply [8] approach to prove global stability of $E^{*}$. Consider the following Lyapunov function

$$
V(S, I, R, B)=\left(S-S^{*} \ln \frac{S}{S^{*}}\right)+M\left(I-I^{*} \ln \frac{I}{I^{*}}\right)+P\left(R-R^{*} \ln \frac{R}{R^{*}}\right)+Q\left(B-B^{*} \ln \frac{B}{B^{*}}\right) .
$$

The derivative of $V$ is

$$
\frac{\mathrm{dV}}{\mathrm{dt}}=\left(1-\frac{S^{*}}{S}\right) \frac{\mathrm{dS}}{\mathrm{dt}}+M\left(1-\frac{I^{*}}{I}\right) \frac{\mathrm{dI}}{\mathrm{dd}}+P\left(1-\frac{R^{*}}{R}\right) \frac{\mathrm{dR}}{\mathrm{dt}}+Q\left(1-\frac{B^{*}}{B}\right) \frac{\mathrm{dB}}{\mathrm{dt}} .
$$

Next, we replace $\frac{\mathrm{dS}}{\mathrm{dt}}, \frac{\mathrm{dI}}{\mathrm{dt}}, \frac{\mathrm{dR}}{\mathrm{dt}}$ and $\frac{\mathrm{dB}}{\mathrm{dt}}$ in Eq. (14) using system (1), to have

$$
\begin{aligned}
\frac{\mathrm{dV}}{\mathrm{dt}}= & \left(1-\frac{S^{*}}{S}\right)\left(\Lambda-\left(\frac{(1-\omega) \alpha B}{\kappa+B}+\mu\right) S\right)+M\left(1-\frac{I^{*}}{I}\right)\left(\frac{(1-\omega) \alpha B S}{\kappa+B}-(\mu+\epsilon+\delta) I\right) \\
& +P\left(1-\frac{R^{*}}{R}\right)(\epsilon I-\mu R)+Q\left(1-\frac{B^{*}}{B}\right)((1-\omega) \eta I-\nu B) .
\end{aligned}
$$

At endemic equilibrium, system (1) becomes 


$$
\begin{aligned}
\Lambda & =\left[\frac{(1-\omega) \alpha B^{*}}{\kappa+B^{*}}+\mu\right] S^{*} \\
(\mu+\epsilon+\delta) & =\frac{(1-\omega) \alpha B^{*} S^{*}}{\left(\kappa+B^{*}\right) I^{*}} \\
\mu & =\frac{\epsilon I^{*}}{R^{*}} \\
\nu & =\frac{(1-\omega) \eta I^{*}}{B^{*}}
\end{aligned}
$$

Substituting (16) into (15), we get

$$
\begin{aligned}
\frac{\mathrm{dV}}{\mathrm{dt}}= & \left(1-\frac{S^{*}}{S}\right)\left(\left[\frac{(1-\omega) \alpha B^{*}}{\kappa+B^{*}}+\mu\right] S^{*}-\left[\frac{(1-\omega) \alpha B}{\kappa+B}+\mu\right] S\right) \\
& +M\left(1-\frac{I^{*}}{I}\right)\left(\frac{(1-\omega) \alpha B S}{\kappa+B}-\frac{(1-\omega) \alpha B^{*} S^{*} I}{\left(\kappa+B^{*}\right) I^{*}}\right) \\
& +P\left(1-\frac{R^{*}}{R}\right)\left(\epsilon I-\frac{\epsilon I^{*} R}{R^{*}}\right)+Q\left(1-\frac{B^{*}}{B}\right)\left((1-\omega) \eta I-\frac{(1-\omega) \eta I^{*} B}{B^{*}}\right),
\end{aligned}
$$

which upon simplification we arrive at

$$
\begin{aligned}
\frac{\mathrm{dV}}{\mathrm{dt}}= & -\mu \frac{\left(S-S^{*}\right)^{2}}{S}+\left(1-\frac{1}{w}\right)\left(\frac{(1-\omega) \alpha B^{*} S^{*}}{\kappa+B^{*}}\right)-\left(1-\frac{1}{w}\right)\left(\frac{(1-\omega) \alpha w z B^{*} S^{*}}{\kappa+z B^{*}}\right) \\
& +M\left(\frac{(1-\omega) \alpha B^{*} S^{*} w z}{\kappa+z B^{*}}-\frac{(1-\omega) \alpha B^{*} S^{*} x}{\left(\kappa+B^{*}\right)}-\frac{(1-\omega) \alpha B^{*} S^{*} w z}{\left(\kappa+z B^{*}\right) x}+\frac{(1-\omega) \alpha B^{*} S^{*}}{\left(\kappa+B^{*}\right)}\right) \\
& +P\left(\epsilon I^{*} x-\epsilon I^{*} y-\frac{\epsilon I^{*} x}{y}+\epsilon I^{*}\right) \\
& +Q\left((1-\omega) \eta I^{*} x-(1-\omega) \eta I^{*} z-\frac{(1-\omega) \eta I^{*} x}{z}+(1-\omega) \eta I^{*}\right)
\end{aligned}
$$

where $w=\frac{S}{S^{*}}, x=\frac{I}{I^{*}}, y=\frac{R}{R^{*}}$ and $z=\frac{B}{B^{*}}$. Furthermore, Eq. (17) can be written as

$$
\frac{\mathrm{dV}}{\mathrm{dt}}=-\mu \frac{\left(S-S^{*}\right)^{2}}{S}+f(w, x, y, z),
$$

where

$$
\begin{aligned}
& f(w, x, y, z)=\left(1-\frac{1}{w}\right)\left(\frac{(1-\omega) \alpha B^{*} S^{*}}{\kappa+B^{*}}\right)-\left(1-\frac{1}{w}\right)\left(\frac{(1-\omega) \alpha w z B^{*} S^{*}}{\kappa+z B^{*}}\right) \\
& +M\left(\frac{(1-\omega) \alpha B^{*} S^{*} w z}{\kappa+z B^{*}}-\frac{(1-\omega) \alpha B^{*} S^{*} x}{\left(\kappa+B^{*}\right)}-\frac{(1-\omega) \alpha B^{*} S^{*} w z}{\left(\kappa+z B^{*}\right) x}+\frac{(1-\omega) \alpha B^{*} S^{*}}{\left(\kappa+B^{*}\right)}\right) \\
& +P\left(\epsilon I^{*} x-\epsilon I^{*} y-\frac{\epsilon I^{*} x}{y}+\epsilon I^{*}\right) \\
& +Q\left((1-\omega) \eta I^{*} x-(1-\omega) \eta I^{*} z-\frac{(1-\omega) \eta I^{*} x}{z}+(1-\omega) \eta I^{*}\right) .
\end{aligned}
$$

To determine $M, P$ and $Q$, we set the coefficients of $w z, y$ and $z$ of Eq. (18) equal to zero and obtain,

$$
\begin{aligned}
& M \frac{(1-\omega) \alpha B^{*} S^{*}}{\kappa+z B^{*}}=0 \\
& -P \epsilon I^{*}=0 \\
& -Q(1-\omega) \eta I^{*}=0
\end{aligned}
$$


Let $\mathrm{M}=1$, solving for $\mathrm{P}$ and $\mathrm{Q}$, we have

$$
P=\frac{(1-\omega) \alpha B^{*} S^{*}}{\left(\kappa+z B^{*}\right) \epsilon I^{*}}, \quad Q=\frac{(1-\omega) \alpha B^{*} S^{*}}{\left(\kappa+z B^{*}\right) \eta I^{*}}
$$

Substituting for $M, P$ and $Q$ in Eq. (18) and simplifying the result, we obtain

$$
\begin{aligned}
f(w, x, y, z)= & \frac{(1-\omega) \alpha B^{*} S^{*}}{\kappa+B^{*}}\left(2-\frac{1}{w}-x\right)+\frac{(1-\omega) \alpha B^{*} S^{*}}{\kappa+z B^{*}}\left(x+z-y-\frac{x}{y}-\frac{w z}{x}+1\right) \\
& +\frac{(1-\omega)^{2} \alpha B^{*} S^{*}}{\kappa+z B^{*}}\left(x-z-\frac{x}{z}+1\right)
\end{aligned}
$$

Using arithmetic and geometric mean inequality, we have

$\frac{1}{w}+x>2, y+\frac{x}{y}+\frac{w z}{x}-x-z>1$ and $z+\frac{x}{z}-x>1$.

Clearly $f(w, x, y, z) \leq 0$, hence $\frac{\mathrm{dG}}{\mathrm{dt}} \leq 0$ in $\Omega$. The equality $\frac{\mathrm{dV}}{\mathrm{dt}}=0$ if and only if $w=x=y=z=1$ and $S=S^{*}, I=I^{*}, R=R^{*}, B=B^{*}$. Thus, system (1) has a unique endemic equilibrium point $E^{*}$ which is globally asymptotically stable if $\mathcal{R}_{0}>1$ using LaSalle's invariance principle in [8].

\section{Results}

We carry out numerical simulations of model (1), using MATLAB ode45 solver. The parameter values used are presented in Table 1.

Figure $2 \mathrm{a}$ shows that in the absence of treatment or presence of ineffective treatment $(0 \leq \omega<1)$, the number of infected individuals decreases at the onset of the disease, followed by a rapid increase and settles at the endemic level. But when treatment is effective $(\omega=1)$, the number of infected individuals decreases and finally converges to the disease-free equilibrium point. Figure $2 \mathrm{~b}$ exhibits that in the absence of treatment or the presence of ineffective treatment $(0 \leq \omega<1)$, the number of pathogens rises rapidly; this is due to the continued shedding of pathogens by infected individuals. Figure $2 \mathrm{~b}$ also shows that when the treatment is effective $(\omega=1)$, the number of pathogens decreases and eventually converges to the disease-free equilibrium point.

Table 1 Parameter values of the model

\begin{tabular}{lll}
\hline Parameter symbol & Value & Source \\
\hline$\Lambda$ & 467 humans/day & {$[9]$} \\
$\mu$ & $0.0247 /$ day & Assumed \\
$\alpha$ & $0.25 /$ day & Assumed \\
$\kappa$ & 50,000 & Assumed \\
$\delta$ & $0.002 /$ day & Assumed \\
$\eta$ & $1.431 \times 10^{-2} /$ day & Assumed \\
$\epsilon$ & $0.0657 /$ day & {$[9]$} \\
$\nu$ & $0.001 /$ day & Assumed \\
$\omega$ & $0 \leq \omega \leq 1$ & Assumed \\
\hline
\end{tabular}




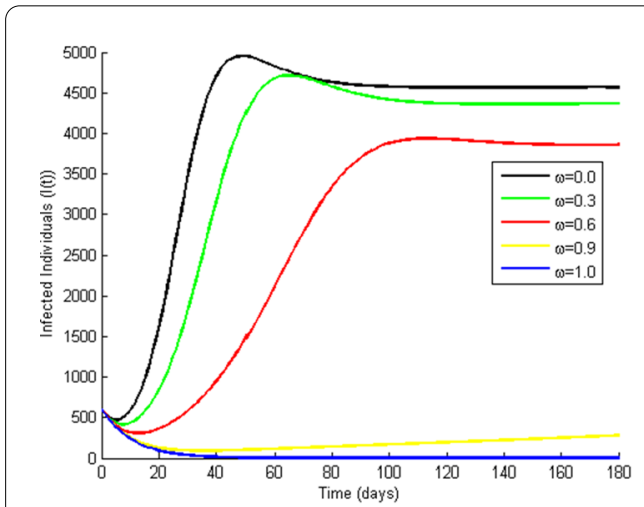

(a)

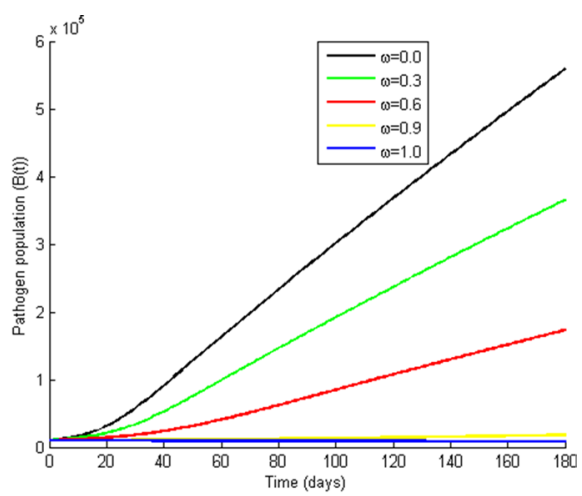

(b)

Fig. 2 The impact of treatment on $\mathbf{a}$ infected individual and $\mathbf{b}$ pathogen population

\section{Conclusion}

In this study, we have formulated a mathematical model describing the transmission of typhoid fever with treatment as control strategy. The model has disease free equilibrium and endemic equilibrium. We have derived the basic reproduction number $\mathcal{R}_{0}$ and shown that the typhoid fever will disappear if $\mathcal{R}_{0}<1$. We have also shown that typhoid fever persists in the population if $\mathcal{R}_{0}>1$, implying that after some period of time the typhoid fever will become hazardous. The findings of the numerical simulation indicate that effective treatment is adequate in eradicating typhoid fever.

\section{Acknowledgements}

The authors appreciate ample time given by their respective universities towards this manuscript.

\section{Author contributions}

This work was carried out in collaboration between both authors. Author HON designed the study and wrote the first draft of the manuscript and performed Mathematical analysis of the study. Author JSM managed literature searches and performed numerical simulation. All authors read and approved the final manuscript.

\section{Funding}

There is no any funding.

Availability of data and materials

Data sharing is not applicable to this paper as no datasets were generated or analyzed during the current study.

\section{Declarations}

\section{Ethics approval and consent to participate}

Authors declare that there is not any ethical approval.

\section{Consent for publication}

Not applicable

\section{Competing interests}

The authors declare that they have no competing interests.

\section{Author details}

${ }^{1}$ Department of Mathematics, The Technical University of Kenya, Nairobi, Kenya. ${ }^{2}$ Department of Mathematics, Laikipia University, Laikipia, Kenya.

Received: 17 November 2020 Accepted: 19 June 2021

Published online: 02 July 2021

\section{References}

1. WHO:Immunization Vaccines and Biologicals. https://www.who.int/teams/immunization-vaccines-and-biologicals/ diseases/typhoid Accessed Accessed 10 Nov 2020 
2. Simiyu, K., Leslie, J.: Typhoid in a Kenyan village: its impact, its prevention. Am. J. Trop. Med. Hygien 95(5), 1112-1113 (2018)

3. CDC:Typhoid Fever Targets Children from Kenyan Urban Slums. https://www.cdc.gov/globalhealth/countries/ kenya/blog/typhoid.htm. Accessed 10 Nov 2020

4. Nthiri, J.K., Lawi, G.O., Akinyi, C.O., Oganga, D.O., Muriuki, W.C., Musyoka, M.J., Otieno, P.O., Koech, L.: Mathematical modelling of typhoid fever disease incorporating protection against infection. Br. J. Math. Comput. Sci. 14(1), 1-10 (2016)

5. Stephen, E., Nyerere, N.: Modelling typhoid fever with education, vaccinatrion and treatment and applied mathematics. Am. J. Trop. Med. Hyg. 2, 156-164 (2016). https://doi.org/10.11648/j.ijtam.20160202.30

6. Temitope, O.O., Oluwatayo, M.O., Segun, N.O., Grace, E.F., Joshua, O.O., Opeyemi, R.A., Omobolaji, Y.H., Adenike, O.O.: On the effect of vaccination, screening and treatment in controlling typhoid fever spread dynamics: deterministic and stochastic applications. Math. Stat. 8(6), 621-630 (2020). https://doi.org/10.13189/ms.2020.080601

7. Van den Driessche, P., Watmough, J.: Reproduction numbers and sub-threshold endemic equilibria for compartmental models of disease transmission. Math. Biosci. 180, 2948 (2002)

8. LaSalle, J.P.:The stability of dynamical systems. In: Regional Conference Series in Applied Mathematics, SIAM, Philadelphia (1976)

9. Mutua, J.M., Wang, F.-B., Vaidya, N.K.: Modeling malaria and typhoid fever co-infection dynamics. Math. Biosci. 264, 128-144 (2015)

\section{Publisher's note}

Springer Nature remains neutral with regard to jurisdictional claims in published maps and institutional affiliations.

\section{Submit your manuscript to a SpringerOpen ${ }^{\circ}$ journal and benefit from:}

- Convenient online submission

- Rigorous peer review

- Open access: articles freely available online

- High visibility within the field

Retaining the copyright to your article

Submit your next manuscript at $>$ springeropen.com 